\title{
Specific Quality of Service Constrained Optimal Allocation of Transmit Power in Uplink Cognitive OFDMA System
}

\author{
Lutfa Akter*, Normin Nahar \\ Department of Electrical and Electronic Engineering, Bangladesh University of Engineering and Technology, \\ Dhaka-1000, Bangladesh. \\ * Corresponding author. Email: lutfaakter@eee.buet.ac.bd \\ Manuscript submitted October 12, 2019; accepted January 10, 2020. \\ doi: 10.17706/ijcce.2020.9.3.105-121
}

\begin{abstract}
This paper investigates an optimal allocation of transmit power for uplink cognitive OFDMA system. The aim is to construct two optimization frameworks namely, framework-I and II for uplink cognitive OFDMA system that minimizes it's transmit power while maintaining Quality of Service (QoS). The measures for QoS include SNR threshold for framework-I whereas, for framework-II, it is measured by minimum rate requirement (bits/sec/Hz) to obtain a certain bit error rate (BER). Simulation results reveal the effectiveness of the proposed frameworks. Additionally, for framework-I, effects of different SNR threshold and users' power budget are observed on the allocation of transmit power. Whereas, for framework-II, effects of different target BER, users' power budget and minimum rate requirement are observed on the allocation of transmit power. Results are also compared with the results obtained from conventional capacity maximization based resource allocation approaches in terms of allocated transmit power, energy efficiency (EE) and spectral efficiency (SE). Simulation results reveal that, the proposed frameworks are incredibly successful in terms of utilization of power budget of users and EE compared to conventional capacity maximization based resource allocation approaches.
\end{abstract}

Key words: Cognitive radio, uplink, OFDMA, transmit power, QoS, SNR, minimum rate requirement, BER, $M$-QAM, EE, SE, resource allocation.

\section{Introduction}

Cognitive radio (CR) is a form of wireless communication in which a transceiver can intelligently detect which communication channels are in use and which are not, and instantly move into vacant channels while avoiding occupied ones. This optimizes the use of available radio-frequency (RF) spectrum while minimizing interference to other users. In cognitive orthogonal frequency division multiple access (OFDMA) scheme, multiple cognitive radios are considered to compete for multiple subcarriers/subchannels. It is to be noted that, cognitive radio is a term proposed by Joseph Mitola III [1] to represent technologies improving spectrum efficiency by adaptive utilization of idle radio resource. In the past few decades enormous research have been carried out regarding cognitive radio technology, hence technological advancement and multiple forms have been shown up in this regard. OFDMA is widely recognized as an ideal air interface for the CR system due to its flexibility in allocating radio resource among the secondary users (SUs), which is the prerequisite for the CR system to acquire high throughput. There has been enormous research on OFDM/OFDMA based cognitive radio system. Most of the prior research efforts on cognitive OFDMA systems are on sensing subchannel [2], [3], channel allocation [4]-[7], subchannel and 
transmit power allocation [8]-[14], transmit power allocation [15]-[22] etc. Most of the approaches on subchannel and transmit power allocation/transmit power allocation [8]-[14] are for downlink systems and based on maximizing capacity criterion which results on full utilization of power budget and hence, incur huge energy consumption. The authors in [15]-[22] study energy efficiency (EE) based transmit power allocation. However, EE based approaches are nonconvex and hence, solutions are near optimal. Power minimization as the optimization criterion is especially desirable in the networks with battery-powered nodes [23]. Although there are few works on power minimization approach in cognitive radio network [23], however the presented approaches are non-convex and a vigorous study is yet to be explored.

In this paper, we tend to develop specific Quality of Service (QoS) constrained power minimization criterion based resource allocation convex optimization framework with transmit power as decision variable considering an uplink cognitive OFDMA system. Here, multiple SUs communicate through multiple subcarriers/subchannels in the considered uplink cognitive OFDMA system. It is assumed that, each subcarrier/subchannel is assigned to a single user only. For such a system, two optimization frameworks are formulatedto determine transmit power based on power minimization criterion. Proposed optimization frameworks are constrained by specific QoS. More specifically, framework-I is constrained by SNR threshold whereas, minimum rate requirement to attain a certain BER is considered as the QoS constraint in framework-II. For both frameworks, the objective function is to minimize transmit power. It is observed that, both of the proposed frameworks are very much successful in terms of minimization of total transmit power and maximization of EE compared to conventional capacity maximization based resource allocation approaches. We also study the effect of variation of transmit power upper bound and other QoS metrics. It is observed that, SNR threshold has a major impact on the performance of framework-I whereas, with the variation in target BER and minimum rate requirement, framework-II shows changes accordingly.

The rest of the paper is organized as follows. The system model is presented in Section 2. The proposed resource allocation framework-I is presented in Section 3, followed by proposed resource allocation framework-II in Section 4. The numerical results of proposed resource allocation framework-I is presented in Section 5, followed by the numerical results of proposed resource allocation framework-II in Section 6 . Section 7 presents the comparison with conventional capacity maximization based resource allocation approach. Finally, Section 8 concludes the paper.

\section{System Model}

An uplink cognitive OFDMA system of $L$ secondary users indexed by $l \in[1 ; 2 ;:: ; L L]$ and $\mathrm{N}$ free subchannels indexed by $n \in[1 ; 2 ;: .: ; N]$ has been considered. All users are considered to be peers to each other. An Access Point (AP) is assumed to control the transmission of cognitive radios that lie within its range of coverage and also collects reports about the activities of primary users (PUs) with whom the CR may interfere with. A common control channel for dialogue between CRs and AP is also assumed.

Overlay spectrum sharing is adopted by secondary users. That is, secondary users use the spectrum when it is unused by PUs. It is assumed that, subchannel allocation to each of the users is already decided by the AP. It is also assumed that, each subcarrier/subchannel is assigned to a single user. An interference temperature (IT) threshold is also imposed to protect the PU transmission on any subchannel from any harmful interference. IT threshold is defined as the total RF power measured at the primary users' receiver antenna per unit bandwidth. As in [24], IT threshold is set to be 200 times of subchannel noise power in this paper. For every user, SNR is computed as:

$$
\gamma_{l, n}=\frac{\alpha_{l, n} p_{l, n} h_{l, l}(n)}{\sigma^{2}(n)}, \forall l, n,
$$


where, $p_{l, n}$ is the transmit power of $l$-th user in $n$-th subchannel, $h_{l, l}(n)$ is the power gain from $l$-th transmitter to $l$-th receiver in $n$-th subchannel, $\alpha_{l, n}$ is the subchannel assignmentindex, $\sigma^{2}(n)$ is the noise power in $n$-th subchannel. As overlay spectrum sharing and orthogonal multiple access scheme is assumed, hence, any SU experiences only subchannel noise. The following assumptions have been implored to enable mathematical tractability of the optimization frameworks:

1) Every active SU radio has an upper bound on total transmit power across subchannels.

2) Simple path loss model for subchannel is considered. More specifically, subchannels are assumed to be independent and identically distributed (IID). The strength of each is assumed to be Rayleigh distributed and thus the power of each subchannel is exponentially distributed.

3) In the first framework, each user has a minimum SNR requirement that needs to be maintained.

4) In the second framework, each user has a minimum rate requirement (bits/sec/Hz) that needs to be maintained.

5) $M$-ary QAM modulation scheme with an adaptive modulation order $M$ is considered.

Under this system model, two optimization frameworks are constructed using the notations in Table 1.

Table 1. Notations

\begin{tabular}{|l|l|}
\hline$\sigma^{2}(n)$ & noise power in $\mathrm{n}$-th subchannel \\
\hline$h_{l, l}(n)$ & $\begin{array}{l}\text { power gain from } l \text {-th transmitter to } l \text {-th receiver in } n \text {-th } \\
\text { subchannel }\end{array}$ \\
\hline$h_{l, n}(m)$ & $\begin{array}{l}\text { power gain from } l \text {-th transmitterat location } \mathrm{m} \text { in } n \text {-th } \\
\text { subchannel }\end{array}$ \\
\hline$p_{l, n}$ & transmit power of $l$-th user in $n$-th subchannel \\
\hline$p^{\text {max }} x_{l, n}$ & maximum transmit power of $l$-th user in $n$-th subchannel \\
\hline$p_{l^{\max }}$ & $\begin{array}{l}\text { maximum transmit Power of } l \text {-th user across it's intended } \\
\text { subchannels }\end{array}$ \\
\hline$I_{t}(n)$ & interference temperature threshold in $n$-th subchannel \\
\hline$\gamma_{l, n}$ & SNR for $l$-th user in $n$-th subchannel \\
\hline$\gamma_{l, n}^{t}$ & SNR threshold at receiver for $l$-th user in $n$-th subchannel \\
\hline$R_{l}$ & achievable rate for $l$-th user in each OFDM subchannel \\
\hline$r_{l, n}$ & achievable rate for $l$-th user in $n$-th subchannel \\
\hline$R_{l}^{\text {min }}$ & minimum rate for $l$-th user \\
\hline$\alpha_{l, n}$ & subchannel assignment index \\
\hline$B E R_{l, n}$ & bit error rate for $l$-th user in $n$-th subchannel \\
\hline$b_{l, n}$ & number of bits per symbol for $l$-th user in $n$-th subchannel \\
\hline
\end{tabular}

\section{Proposed Resource Allocation Optimization Framework-I}

In this paper, the transmit power of secondary user is optimized. Framework-I is constructed constituting the objective function to be optimized and the constraints that have to be fulfilled. These can be represented as:

Determine $\mathbf{p}=\left[p_{1,1}, \ldots, p_{L, 1}, \ldots, p_{1, \mathrm{~N}}, \ldots, p_{L, N}\right]^{T}$

To Minimize

$$
F=\sum_{\mathrm{n}=1}^{N} \sum_{l=1}^{L} p_{l, n}
$$

Subject to 


$$
\begin{gathered}
C_{11}: \sum_{n=1}^{N} p_{l, n} \alpha_{l, n} \leq p_{l}^{\max }, \quad \forall l \\
C_{12}: p_{l, n} h_{l, n}(m) \alpha_{l, n} \leq I_{t}(n), \quad \forall l, n \\
C_{13}: \gamma_{l, n} \geq \gamma_{l, n}^{t}, \quad \forall l, n
\end{gathered}
$$

where,

$$
\gamma_{l, n}=\frac{\alpha_{l, n} p_{l, n} h_{l, l}(n)}{\sigma^{2}(n)}, \forall l, n \quad p_{l, n} \geq 0, \forall l, n
$$

Here, $C_{11}$ indicates limit on total transmit power of user $l$ across its intended subchannels; $C_{12}$ indicates the interference temperature constraint and $C_{13}$ indicates the SNR constraint required to guarantee desired QoS. The subchannel assignment index $\alpha_{l, n}$ is given as:

$$
\begin{aligned}
\alpha_{l, n} & =1, \text { subchannel } \mathrm{n} \text { is allocated to user } l \\
& =0 \text {, other wise }
\end{aligned}
$$

This is a convex optimization problem. The convexity of QoS/SNR constraint $\left(\mathrm{C}_{13}\right)$ is discussed in Theorem 1.

Solution to this optimization problem provides optimal transmit power that every secondary user needs to use in the subchannel that they are operating in.

Theorem 1. $\quad \gamma_{l, n} \geq \gamma_{l, n}^{t}, \forall l, n, \quad$ is a convex constraint:

Proof. From Eq. (1) and (2) the constraint can be rewritten as:

$$
\alpha_{l, n} p_{l, n} h_{l, l}(n) \geq \gamma_{l, n}^{t}\left(\sigma^{2}(n)\right)
$$

Eq. (4) is equivalent to:

$$
\gamma_{l, n}^{t}\left(\sigma^{2}(n)\right)-\alpha_{l, n} p_{l, n} h_{l, l}(n) \leq 0
$$

This inequality is a linear combination of variables. Hence, the inequality is linear, can be treated as convex.

\section{Proposed Resource Allocation Optimization Framework-II}

In this optimization framework, one of the considerations is to ensure minimum rate requirement as a measure of QoS. In order to model such consideration, each user rate $R_{l}$ needs to be greater than minimum rate $R_{l}^{\text {min. }}$. In this context, QoS is defined in achieving rates no less than $R_{l}^{\text {min }}$ with $B E R_{l, n} \leq \zeta$ in all subchannels. Assuming a target BER equals to $\zeta$, the mathematical description of the proposed optimization problem corresponds to:

Determine $\mathbf{p}=\left[p_{1,1}, \ldots, p_{L, 1}, \ldots, p_{1, \mathrm{~N}}, \ldots, p_{L, N}\right]^{T}$

To Minimize

$$
F=\sum_{\mathrm{n}=1}^{N} \sum_{l=1}^{L} p_{l, n}
$$

Subject to

$$
\begin{gathered}
C_{21}: \sum_{n=1}^{N} p_{l, n} \alpha_{l, n} \leq p_{l}^{\max }, \quad \forall l \\
C_{22}: p_{l, n} h_{l, n}(m) \alpha_{l, n} \leq I_{t}(n), \quad \forall l, n
\end{gathered}
$$




$$
C_{23}: R_{l}=\sum_{n=1}^{N} r_{l, n}=\sum_{n=1}^{N} \alpha_{l, n} \log _{2}\left(1+b_{3} \frac{p_{l, n} h_{l, l}(n)}{\sigma^{2}(n)}\right) \geq R_{l}^{\min }, \quad \forall l
$$

where, $b_{3}$ is a constant whose computation is given in the description below and

$$
p_{l, n} \geq 0, \forall l, n
$$

Constraints $C_{21}$ and $C_{22}$ remain same to the constraints as described in framework-I. Constraint $C_{23}$ indicates the achievable rate for $l$-th user and is the sum of the rates in each OFDM subchannel as mentioned earlier. The subchannel assignment index $\alpha_{l, n}$ is already described in framework-I. Using the approximate formulation of BER for $M$-QAM [25], it can be stated that:

$$
B E R_{l, n} \approx b_{1} \exp \left[\frac{-b_{2} \gamma_{l, n}}{2^{b_{l, n}-1}}\right]
$$

where, $b_{1}$ and $b_{2}$ are constants, The approximation is tight within $1 \mathrm{~dB}$ for $b_{l, n} \geq 2$ and $B E R_{l, n} \leq 10^{-3}$.

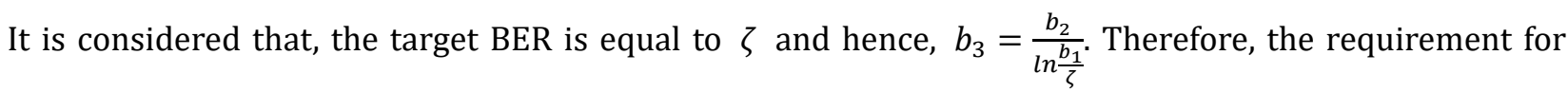
the desired BER to achieve a certain level of QoS is considered in definition of the rate in Eq. (8). This is a convex optimization problem. The convexity of QoS/Minimum rate requirement constraint $\left(C_{23}\right)$ is discussed in Solution to this optimization problem provides optimal transmit power that every secondary user needs to use in the subchannel that they are operating in.

Theorem 2, $\quad R_{l} \geq R_{l}^{\min }, \forall l \quad$ is a convex constraint:

Proof. From Eq. (6) the constraint is rewritten as:

$$
\sum_{n=1}^{N} \alpha_{l, n} \log _{2}\left(1+b_{3} \frac{p_{l, n} h_{l, l}(n)}{\sigma^{2}(n)}\right) \geq R_{l}^{\min }, \forall l
$$

Eq. (9) is equivalent to:

$$
\begin{gathered}
R_{l}^{\text {min }}-\sum_{n=1}^{N} \alpha_{l, n} \log _{2}\left(1+b_{3} \frac{p_{l, n} h_{l, l}(n)}{\sigma^{2}(n)}\right) \leq 0, \quad \forall l \\
o r, R_{l}^{\text {min }}-\alpha_{l, 1} \log _{2}\left(1+b_{3} \frac{p_{l, l} h_{l, l}(1)}{\sigma^{2}(1)}\right)- \\
\ldots-\alpha_{l, n} \log _{2}\left(1+b_{3} \frac{p_{l, n} h_{l, l}(n)}{\sigma^{2}(n)}\right)- \\
\ldots-\alpha_{l, N} \log _{2}\left(1+b_{3} \frac{p_{l, N} h_{l, l}(N)}{\sigma^{2}(N)}\right) \leq 0, \quad \forall l
\end{gathered}
$$

Now, a single term $-\alpha_{l, n} \log _{2}\left(1+b_{3} \frac{p_{l, n} h_{l, l}(n)}{\sigma^{2}(n)}\right)$ is considered and it is denoted as $f$. The first derivative is obtained as:

$$
\frac{d f}{d p_{l, n}}=-\alpha_{l, n} \frac{b_{3} h_{l, l}(n)}{\ln (2)\left(1+b_{3} p_{l, n} h_{l, l}(n)\right)}
$$

Finally, the second derivative is obtained as:

$$
\frac{d^{2} f}{d p_{l, n}^{2}}=\frac{\alpha_{l, n}\left(b_{3} h_{l, l}(n)\right)^{2}}{\ln (2)\left(1+b_{3} p_{l, n} h_{l, l}(n)\right)^{2}}
$$

The constraint described in Eq. (9) represents an inequality. The second term of the inequality is shown as a series in Eq. (10). If a single term of the series is studied it is evident that, the term represents a function of Logarithm. From Eq. (12), it can be seen that, the outcome of the second order derivative of the 
function is obtained as a positive term, hence the function can be treated as convex. As the constraint $C_{3}$ described in Eq. (9) is a combination of such convex functions, it can also be treated as convex.

The energy efficiency (EE) of the system is defined as the ratio of total system throughput to total system power and expressed as:

$$
E E=\frac{R_{S}}{\sum_{n=1}^{N} \sum_{l=1}^{L} \alpha_{l, n} p_{l, n}}, \forall l, n \ldots
$$

where,

$$
R_{S}=\sum_{n=1}^{N} \sum_{l=1}^{L} \alpha_{l, n} \log _{2}\left(1+\frac{p_{l, n} h_{l, l}(n)}{\sigma^{2}(n)}\right), \forall l, n
$$

Here, $R_{S}$ is the system throughput. The spectral efficiency (SE) of the system can be expressed as:

$$
S E=R_{S}
$$

\section{Numerical Results of Proposed Framework-I}

The performance of the optimization framework is quantified for 8 opportunistic users allocated to 64 subchannels in the wireless channel. Each secondary user can be allocated to more than one subchannel. The number of subchannels to which the allocation is performed can be found in Table 2, when the allocated subchannels to each user are listed in Table 3. Table 4 lists the total transmit power upper bound of all SUs for proposed optimization framework-I. Table 5 provides all other system parameters that are required for optimization framework-I.

Table 2. Number of Subchannels Allocated to Users

\begin{tabular}{|c|c|c|c|c|c|c|c|c|}
\hline Users & 1 & 2 & 3 & 4 & 5 & 6 & 7 & 8 \\
\hline Total number of subchannel used & 4 & 7 & 5 & 11 & 12 & 8 & 10 & 7 \\
\hline
\end{tabular}

Table 3. Allocated Subchannels to Each User

\begin{tabular}{|l|l|l|l|l|l|l|l|l|l|l|l|l|}
\hline SU1 & 2 & 27 & 42 & 46 & & & & & & & & \\
\hline SU2 & 6 & 7 & 8 & 14 & 19 & 26 & 54 & & & & & \\
\hline SU3 & 20 & 23 & 25 & 33 & 49 & & & & & & & \\
\hline SU4 & 1 & 4 & 13 & 35 & 38 & 44 & 46 & 53 & 56 & 57 & 59 & \\
\hline SU5 & 5 & 11 & 17 & 21 & 34 & 36 & 39 & 40 & 43 & 45 & 55 & 63 \\
\hline SU6 & 10 & 22 & 41 & 48 & 51 & 60 & 61 & 62 & & & & \\
\hline SU7 & 3 & 9 & 12 & 15 & 24 & 30 & 37 & 50 & 52 & 64 & & \\
\hline SU8 & 16 & 18 & 28 & 29 & 31 & 32 & 58 & & & & & \\
\hline
\end{tabular}

Table4. Total Transmit Power Upper Bound of Users for Framework-I

\begin{tabular}{|l|l|l|l|l|l|l|l|l|}
\hline User, $l$ & 1 & 2 & 3 & 4 & 5 & 6 & 7 & 8 \\
\hline$P_{l, n}$ (Watt) & 4 & 7 & 5 & 11 & 12 & 8 & 10 & 7 \\
\hline
\end{tabular}

Table 5. System Parameters for Framework-I

\begin{tabular}{|c|c|}
\hline$\sigma^{2}(n)($ Watt $)$ & $1.5\left(\times 10^{-10}\right)$ \\
\hline$\gamma_{l, n}^{t} \forall l, n(d B)$ & 10 \\
\hline$I_{t}(n) \forall l, n($ Watt $)$ & $200 \times \sigma^{2}(n)$ \\
\hline
\end{tabular}

With this simulation setup, the performance of the proposed optimization framework is observed. 


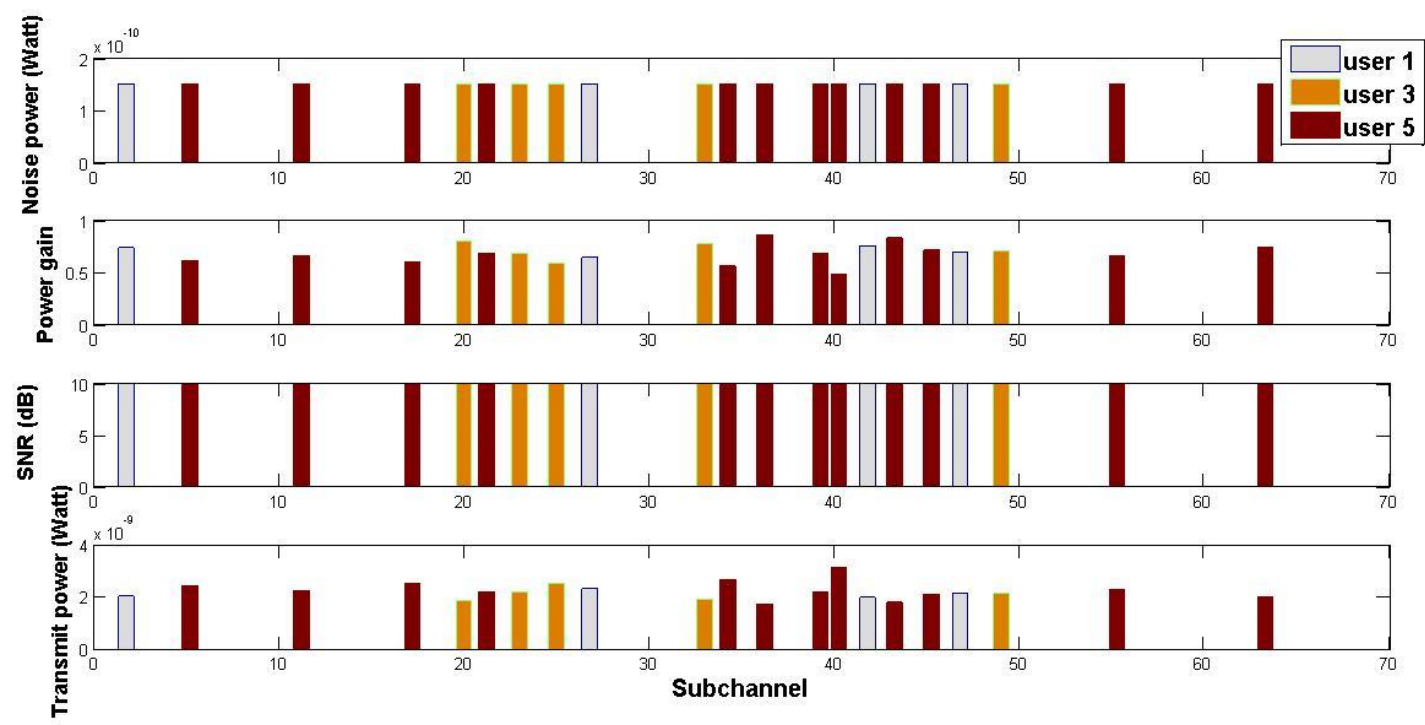

Fig. 1. Allocation of transmit power and resulting SNR.

Simulation 01: First, the performance of proposed framework-I is observed in terms of the allocated transmit power and obtained SNR across the subchannels. For the sake of comparison and clarity, only the allocation of transmit power for users 1, 3 and 5 are observed in different subchannels along with the SNR experienced by the users in respective subchannels. Fig. 1 depicts the allocated transmit power and resulting SNR across subchannels for user 1; 3; 5.The subchannel noise power and subchannel power gain are also shown in the same figure. From Fig. 1, it is reasonably lucid that, the users require to transmit with more power in a subchannel with smaller power gain and vice versa. Same SNR is achieved in all subchannels which is equal to the threshold. So, it is seen that, framework-I allocates transmit power just to attain the SNR threshold. This is due to the objective function of the framework. Hence, successful satisfaction of the QoS constraint of SNR threshold through the framework is marked. Similar pattern is obtained for all other users as well.

The total allocated transmit power across users obtained by proposed framework-I is shown in Fig. 2 . From Fig. 2, it is observed that, the total power spent is within the users' upper limit. That is, framework-I does not spend all of its power as in existing frameworks based on capacity maximization.

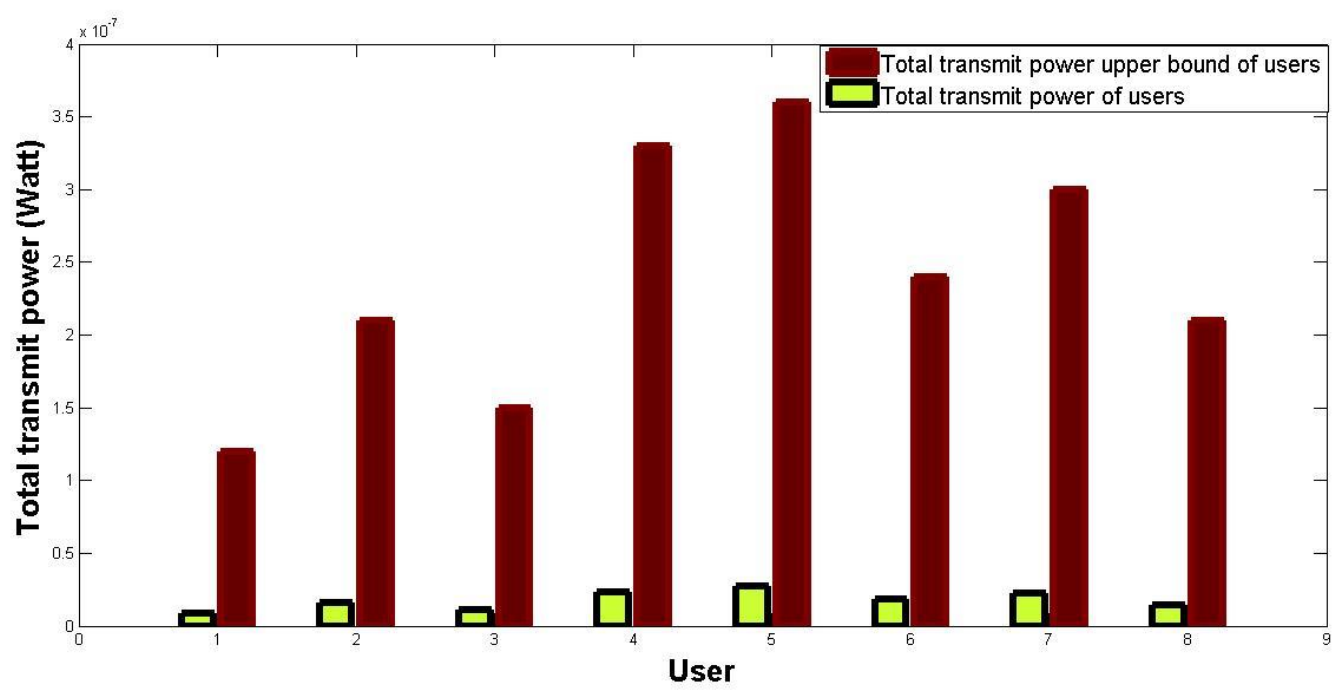

Fig. 2. Allocation of total transmit power across users. 


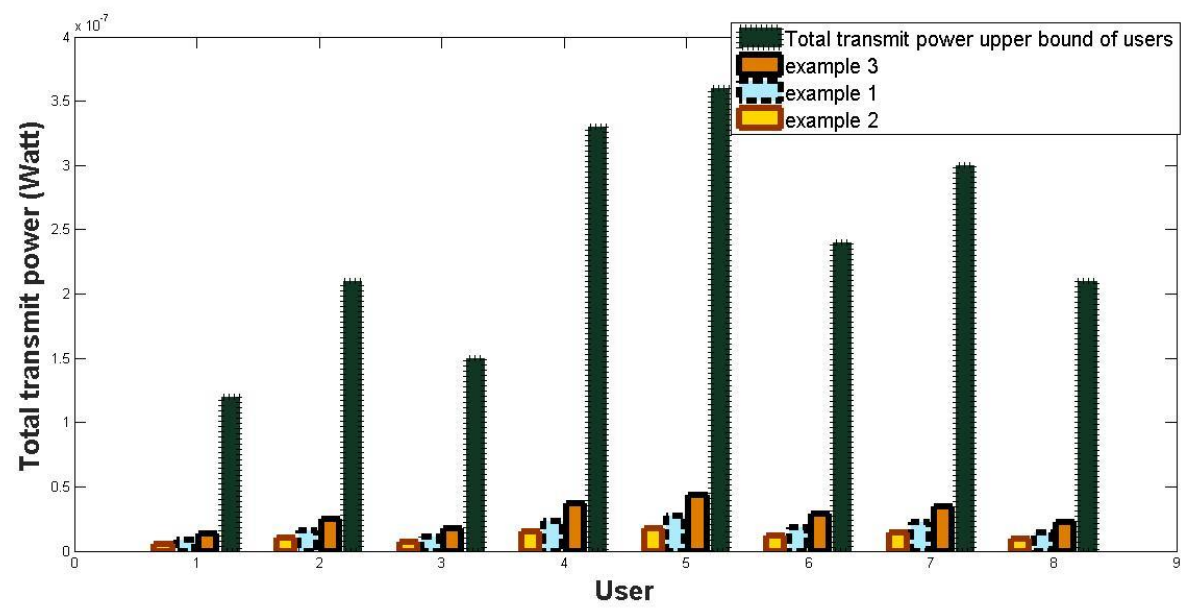

Fig. 3. Allocation of total transmit power across users for different SNR thresholds.

Simulation 02: If the threshold value of the QoS constraint of SNR is changed, it may affect the allocation of transmit power which is a field of interest and hence, the effect is studied next. The SNR threshold shown in Table 5 is considered as example 1. Next the SNR threshold is set to $8 \mathrm{~dB}$ and termed as example 2 . Whereas, the SNR threshold is set to $12 \mathrm{~dB}$ and termed as example 3. Other system parameters are same as in Simulation 01. Fig. 3 shows that, with the increase in the value of SNR threshold, total transmit power across users also increases and vice versa. This is because, to achieve higher SNR threshold and to provide users with it, transmit power increases. However in every cases, it remains within the limit of total transmit power upper bound of users. Which means total transmit power across users is proportional to the SNR threshold which is clearly shown in Fig. 3. It is to be noted that, successful satisfaction of total transmit power upper bound of users is marked for all three example cases.

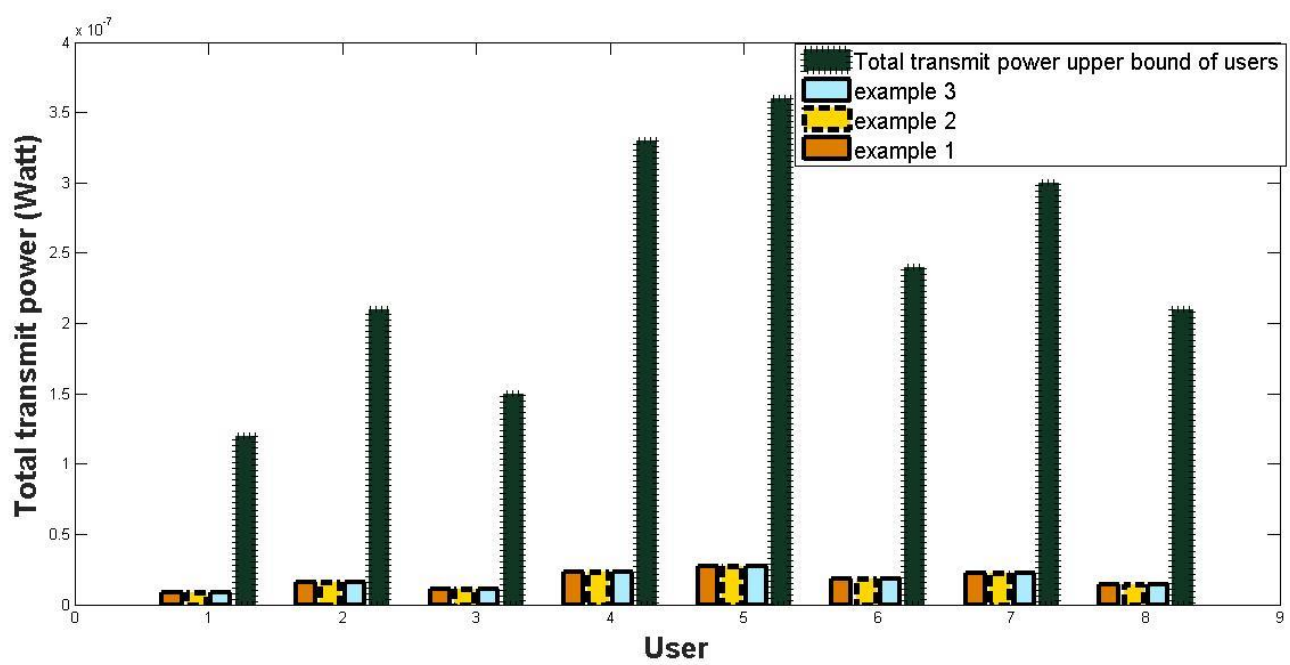

Fig. 4. Allocation of total transmit power across users for different users' power budget.

Simulation 03: Power budget in the delineated framework restricts a secondary user's total budget of transmit power, the impact of changing which is studied in Fig. 4. Here, total transmit power upper bound of users shown in Table 4 is considered as example 1 and the total transmit power upper bound of users shown in Table 6 and Table 7 are considered as example 2 and example 3, respectively. Other system parameters are same as in Simulation 01. Fig. 4 shows the allocation of total transmit power across users for different users' power budget. Fig. 4 shows that, total transmit power for all three examples are identical. 
Hence, as long as QoS constraints of the users are invariable, changing the users' power budget does not have any significant impact on the performance on the proposed framework-I in terms of total transmit power of users once the optimal transmit power is achieved. Once again, it is observed that, total transmit power in every case remains within the limit of total transmit power upper bound of users.

Table 6. Total Transmit Power Upper Bound of Users for Example

\begin{tabular}{|l|l|l|l|l|l|l|l|l|}
\hline User, $l$ & 1 & 2 & 3 & 4 & 5 & 6 & 7 & 8 \\
\hline$P_{l, n}($ Watt $)$ & 6 & 10.5 & 7.5 & 16.5 & 18 & 12 & 15 & 10.5 \\
\hline
\end{tabular}

Table 7. Total Transmit Power Upper Bound of Users for Example 3

\begin{tabular}{|l|l|l|l|l|l|l|l|l|}
\hline User, $l$ & 1 & 2 & 3 & 4 & 5 & 6 & 7 & 8 \\
\hline$P_{l, n}($ Watt $)$ & 2 & 3.5 & 2.5 & 5.5 & 6 & 4 & 5 & 3.5 \\
\hline
\end{tabular}

\section{Numerical Results of Proposed Framework-II}

Table 8 lists the total transmit power upper bound of all SUs for proposed framework-II. Table 9 illustrates the minimum rate (bits/sec/Hz) requirement to achieve a targeted BER for all users. Table 10 provides all other system parameters that are required for proposed optimization framework-II. subchannel allocation and power gain of users will remain same as framework-I. With this simulation setup, the performance of the proposed optimization framework is observed.

Table 8. Total Transmit Power Upper Bound of Users for Framework-II

\begin{tabular}{|l|l|l|l|l|l|l|l|l|}
\hline User, $l$ & 1 & 2 & 3 & 4 & 5 & 6 & 7 & 8 \\
\hline$P_{l, n}($ Watt $)$ & 10 & 10 & 10 & 10 & 10 & 10 & 10 & 10 \\
\hline
\end{tabular}

Table 9. Minimum Rate (Bits/Sec/Hz) Requirement of Users for Framework-II

\begin{tabular}{|l|l|l|l|l|l|l|l|l|}
\hline User, $l$ & 1 & 2 & 3 & 4 & 5 & 6 & 7 & 8 \\
\hline$R_{l}{ }^{\text {min }}$ (bits/sec/Hz) & 11 & 1.1 & 1.4 & 1.8 & 0.5 & 1.7 & 1.4 & 1.9 \\
\hline
\end{tabular}

Table 10. System Parameters for Framework-II

\begin{tabular}{|c|c|}
\hline$\sigma^{2}(n)($ Watt $)$ & $1.5\left(\times 10^{-10}\right)$ \\
\hline$B E R_{l, n}$ & $10^{-3}$ \\
\hline$b_{3}$ & 0.283 \\
\hline$I_{t}(n) \forall l, n($ Watt $)$ & $200 \times \sigma^{2}(n)$ \\
\hline
\end{tabular}

Simulation 01: First, the performance of proposed framework-II in terms of the allocated transmit power and resulting SNR across the subchannels for SU-1, 3 and 5 are observed. Fig. 5 depicts the transmit power and resulting SNR across subchannels for user $1 ; 3 ; 5$. The subchannel noise power and subchannel power gain are also shown in the samefigure. From Fig. 5 , it is seen that, transmit power shows no change with the variation of power gain across subchannel. Here, all users required to transmit with same power across the subchannels. That means, transmit power across subchannel does not have any major relation with subchannel power gain and hence, remains unchanged for all users while keeping noise power constant across subchannels. However, observation reveals that, obtained SNR is proportional to power gain of the subchannels. That means, resulting SNR in a transmission is more when subchannel power gain is higher and vice versa. Similar pattern is obtained for all other users as well. 


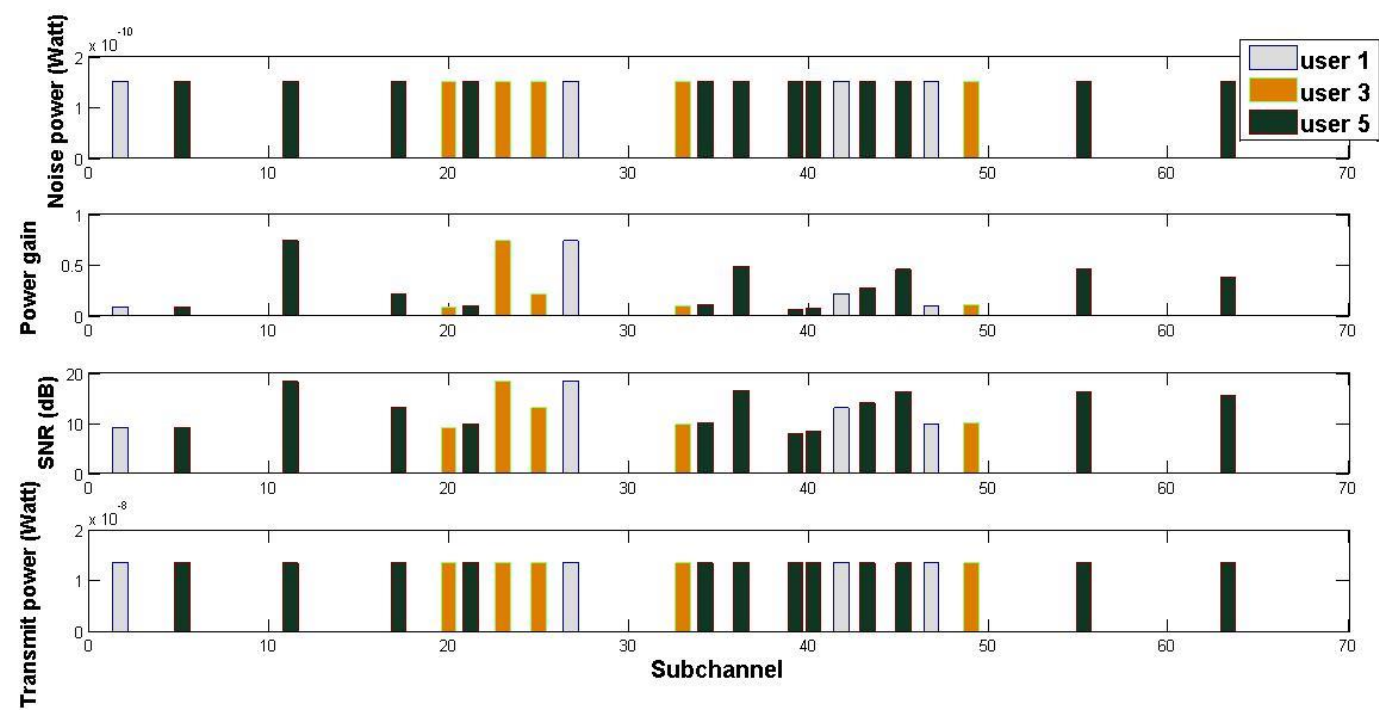

Fig. 5. Allocation of transmit power and resulting SNR.
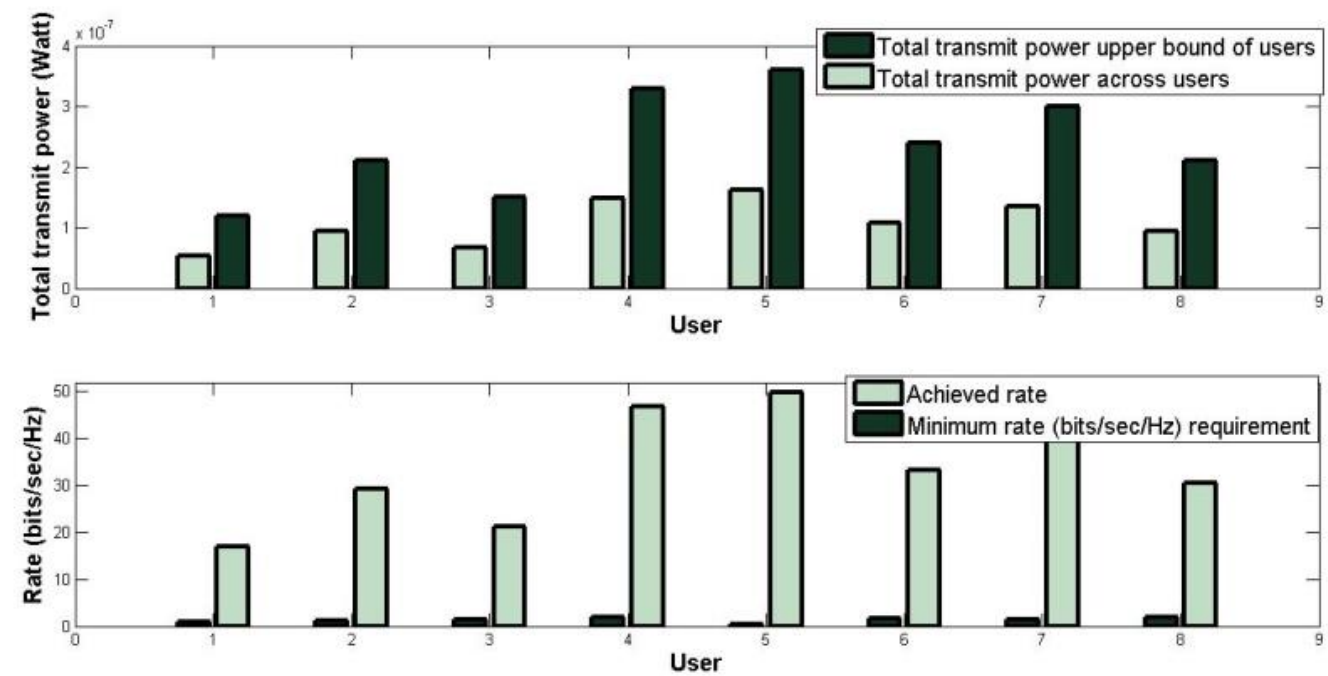

Fig. 6. Allocation of total transmit power and achieved rate (bits/sec/Hz) across users.

Total allocated transmit power across users obtained by proposed framework-II is shown in Fig. 6. It is observed that, the total power spent is within the users' upper limit. That is, just like framework-I, framework-II also does not spend all of it's power as in existing frameworks based on capacity maximization. To ensure optimality of solution, as minimum rate (bits $/ \mathrm{sec} / \mathrm{Hz}$ ) requirement constraint to obtain a certain BER of proposed optimization framework has been considered, hence it is also important to see whether the used bits/sec/Hz constraint is satisfying the minimum requirement of the users. Fig. 6 also shows the total bits/sec/Hz use across users. Minimum rate (bits/sec/Hz) requirement for all the users is also depicted in the same figure. From this figure it can be seen that, proposed optimization framework-II is successful in satisfying both the total transmit power upper bound and minimum bits $/ \mathrm{sec} / \mathrm{Hz}$ use requirement, $R_{l}^{\min }$ for all users.

Simulation 02: As minimum rate requirement (bits/sec/Hz) to obtain a certain BER is a QoS constraint in framework-II, effect of changing the value of BER is another point of interest and can be inspected. Fig. 7 presents the allocation of total transmit power and achieved rate (bits/sec/Hz) across users for two different BER, BER $\mathrm{l}_{\mathrm{ln}}$ values, which are termed as example 1 and example 2, respectively. Total transmit power upper bound and minimum rate (bits/sec/Hz) requirement for users are also depicted in Fig. 7. Here, 
$\mathrm{BER}_{\mathrm{ln}}$ is set to 10 al 3 and we term it as example 1 while, for example $2, \mathrm{BER}_{\mathrm{ln}}$ is set to $10^{-6}$. The value of $b_{3}$ is computed following the method discussed earlier. $b_{3}=0.283$ is considered to guarantee a BER that is less than or equal to $10^{-3}$ while b3 is set to 0.1129 to guarantee a BER that is less than or equal to $10^{-6}$. It is to be noted that, smaller BER means better performance. Other system parameters are same as in Simulation 01.

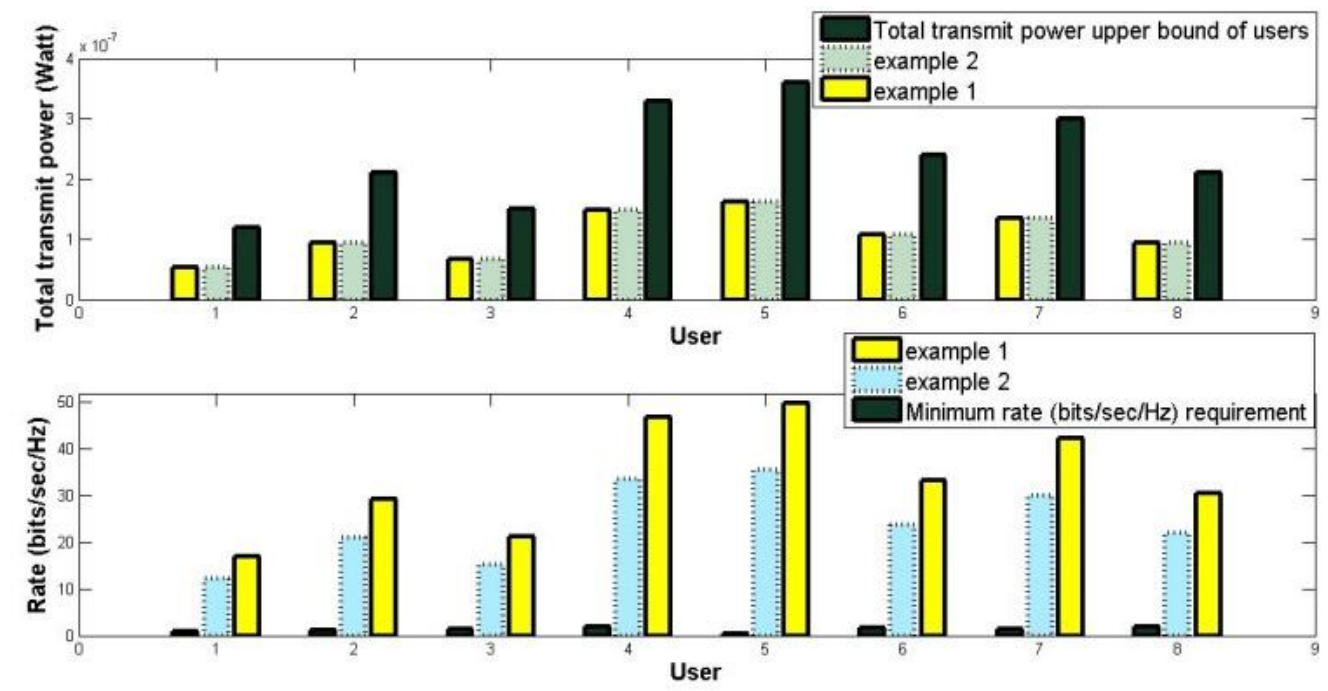

Fig. 7. Allocation of total transmit power and achieved rate (bits/sec/Hz)across users for different target BER.

It is observed that, with the variation in target BER, total transmit power across users shows no changes. Which means, total transmit power for example 2 remains identical to the one achieved in example 1 . Hence, it is seen that, target BER does not have any significant impact on the performance of the proposed framework in terms of total transmit power of users once optimal transmit power is achieved. From the figure it is obvious that, the proposed optimization framework-II is successful in satisfying the total transmit power upper bound of users in both examples. Further observation reveals that, with the variation in target BER, achieved rate (bits/sec/Hz) across users show changes accordingly. That is, achieved rate is higher when target BER is higher and vice versa, which is logical. Although, achieved rate for example 1 is higher compared to example 2, but the proposed optimization framework-II is successful in satisfying the minimum rate requirement for all users in both examples.

Simulation 03: Now, the impact of users' minimum rate (bits/sec/Hz) requirement on the performance of the proposed optimization framework-II is observed. Here, minimum rate requirement shown in Table 9 is considered as example 1and the minimum rate requirement of users shown in Table 11 is considered as example 2. Other system parameters are same as in Simulation 01. Fig. 8 shows the allocation of total transmit power and achieved rate (bits/sec/Hz) across users for examples 1 and 2. Fig. 8 shows that, total transmit power for example 2 increases compared to example 1 but in both the cases, it remains within the total transmit power upper bound of users. Hence, increased value of users' minimum rate requirement has significant impact on the performance on the proposed framework in terms of total transmit power. It is observed that, for both the examples, total transmit power allocation is obtained with in the upper limit of users, hence, proposed framework-II performs successfully with in total transmit power upper bound of users. Minimum rate requirement of users for both examples are also depicted in the same figure. From Fig. 8 it is seen that, with the variation of users' minimum rate requirement, achieved rate also shows obvious changes. It is evident that, achieved rate is more when the minimum rate requirement is higher but in every cases it satisfies respective minimum rate requirement of users. That means, the proposed optimization 
framework is successful in satisfying the minimum rate requirement for all users in every case.

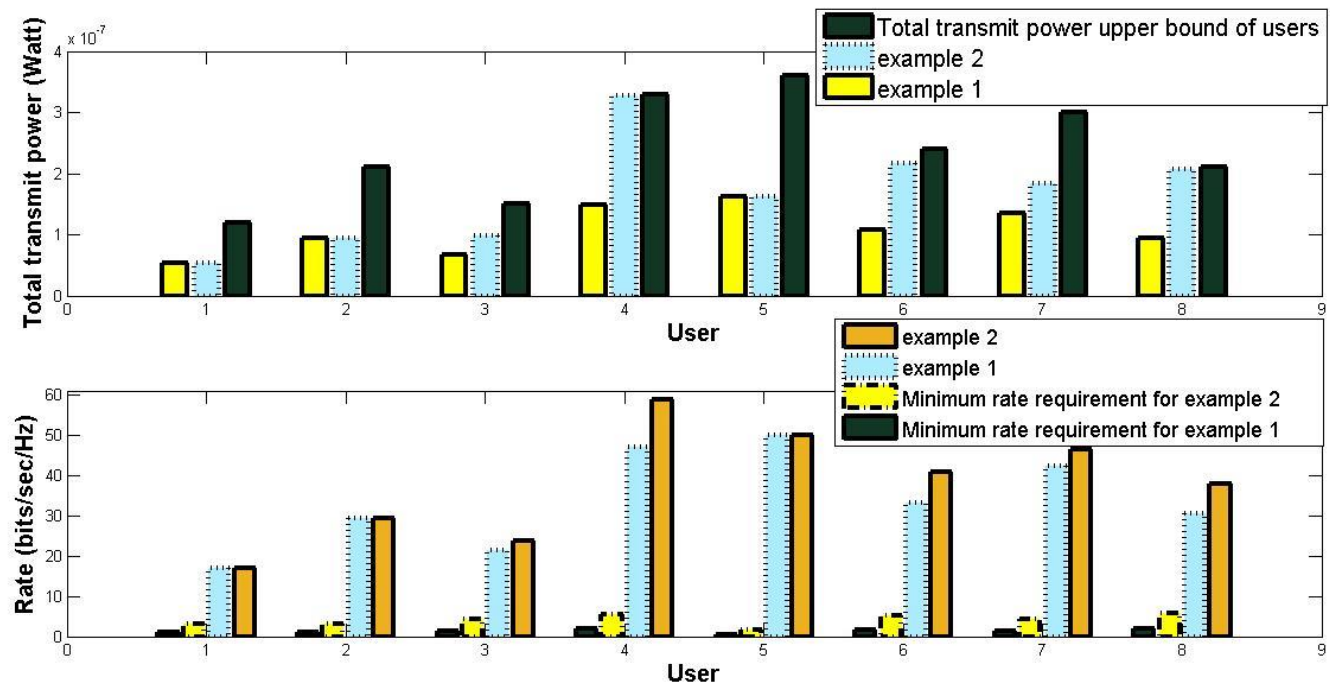

Fig. 8. Allocation of total transmit power and achieved rate (bits/sec/Hz)across users for different Users' minimum rate requirement.

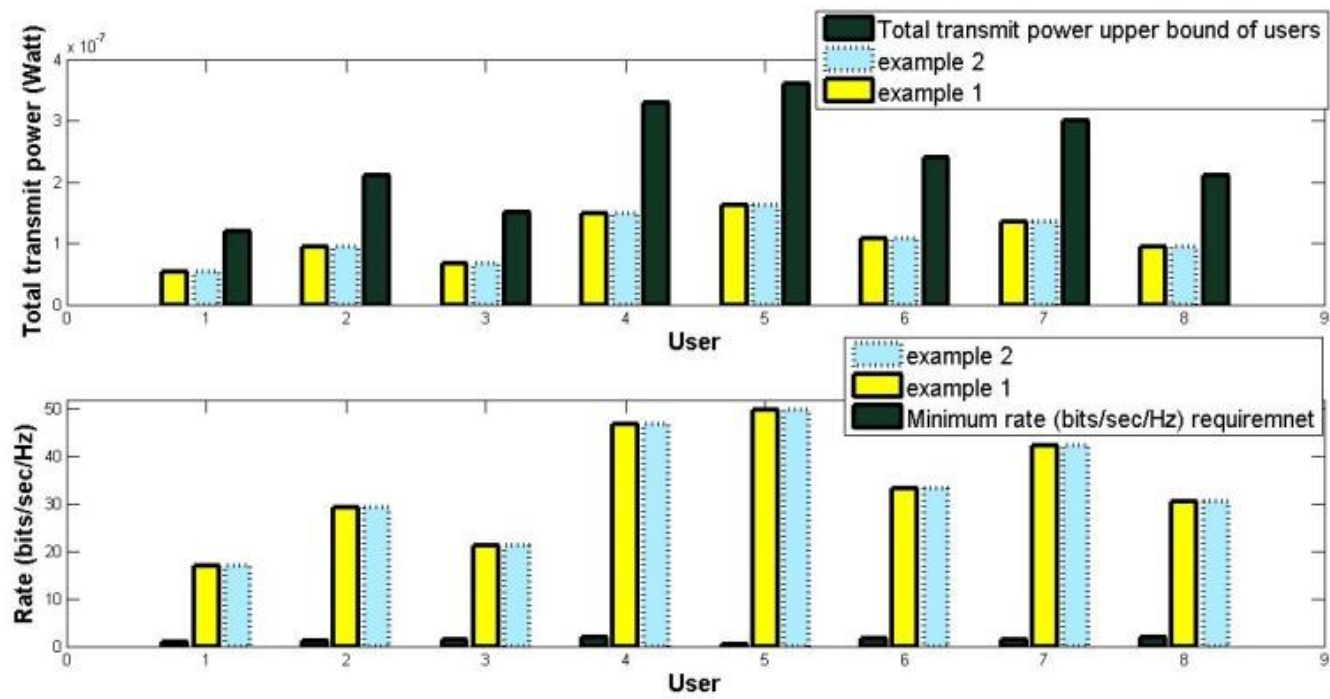

Fig. 9. Allocation of total transmit power and achieved rate (bits/sec/Hz)across users for different Users' power budget.

Simulation 04: Now, the impact of users' power budget on the performance of the proposed optimization framework-II is observed. Here, total transmit power upper bound of users shown in Table 8 is considered as example 1 and the total transmit power upper bound of users shown in Table 12 is considered as example 2. Other system parameters are same as in Simulation 01. Fig. 9 shows the allocation of total transmit power and achieved rate (bits/sec/Hz) across users for both example 1 and 2. Fig. 9 also shows the total transmit power upper bound and minimum rate requirement of users. Fig. 9 shows that, total transmit power across users for both the examples are identical. The study points that, as long as QoS constraints of the users are invariable, increasing the total power budget has no effect on the allocation of transmit power and hence, users' power budget does not have any significant impact on the performance on the proposed framework-II. It is also observed that, with the increased value of users' power budget, achieved rate shows 
no changes. Which means both total transmit power and achieved rate for example 2 is identical to the one achieved in example 1 and hence the proposed optimization framework is successful in satisfying both total transmit power upper bound and minimum rate requirement for all users.

Table 11. Minimum Rate (Bits/Sec/Hz) Requirement of Users for Example 2

\begin{tabular}{|l|l|l|l|l|l|l|l|l|}
\hline User, $l$ & 1 & 2 & 3 & 4 & 5 & 6 & 7 & 8 \\
\hline$R_{l,}{ }^{\min }(\mathrm{bits} / \mathrm{sec} / \mathrm{Hz})$ & 3 & 3.1 & 4.2 & 5.4 & 1.5 & 5.1 & 4.2 & 5.7 \\
\hline
\end{tabular}

Table 12. Total Transmit Power Upper Bound of Users for Example 2

\begin{tabular}{|l|l|l|l|l|l|l|l|l|}
\hline User, $l$ & 1 & 2 & 3 & 4 & 5 & 6 & 7 & 8 \\
\hline$P_{l, n}($ Watt $)$ & 15 & 15 & 15 & 15 & 15 & 15 & 15 & 15 \\
\hline
\end{tabular}

\section{Comparison with Resource Allocation Approach Based on Capacity Maximization}

Following conventional capacity maximization based approach [8], the objective function in optimization frameworks shown in Eqs. (1) and (6) are replaced by:

To Maximize

$$
F=\sum_{n=1}^{N} \sum_{l=1}^{L} \alpha_{l, n} \log _{2}\left(1+\frac{p_{l, n} h_{l, l}(n)}{\sigma^{2}(n)}\right)
$$

Fig. 10 illustrates total transmit power across users obtained from framework-I and II. Total transmit power across users obtained from capacity maximization approach is also shown in the same figure. For proposed framework-I and II, it is seen that, total power spent is with $\mathrm{n}$ the users' upper limit. That is, proposed frameworks do not spend all of their power. Whereas, existing capacity maximization based approach spends all its power which is very obvious from the figure. Hence, proposed frameworks are very successful in terms of utilization of power budget.

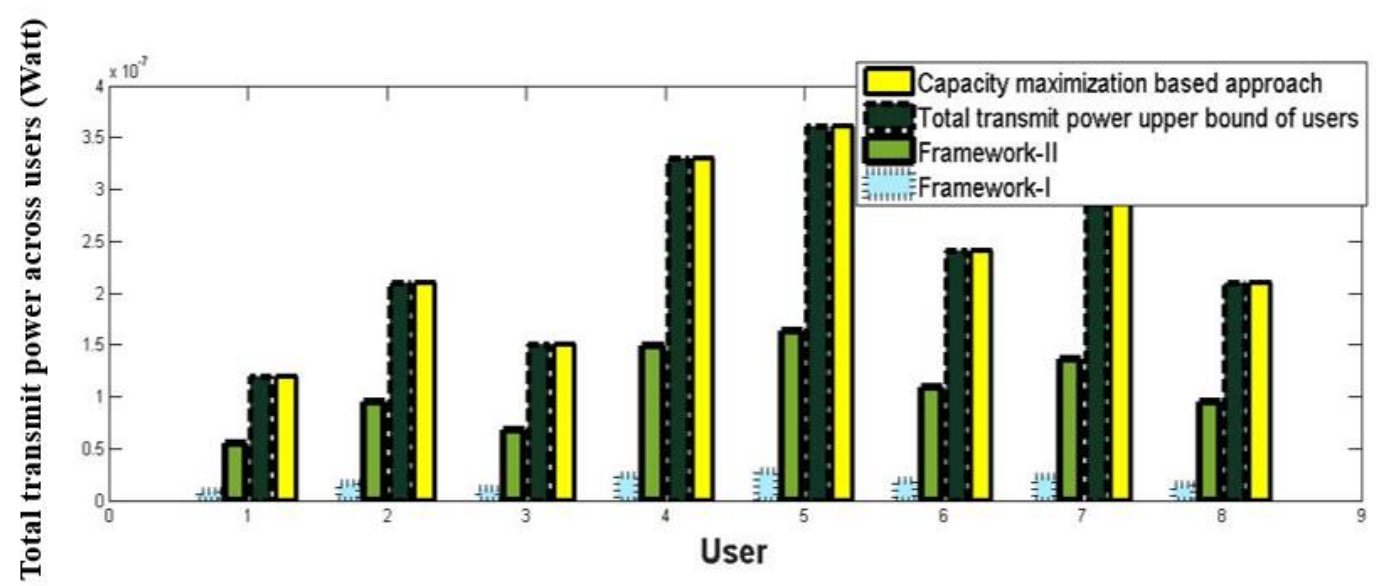

Fig. 10. Allocation of total transmit power across users.

Next, another field of interest is spectral efficiency of the frameworks. From Fig. 11 it is clearly observed that, capacity maximization based approach tends to be more spectral efficient compared to the proposed frameworks. At the same time, framework-II presents more spectral efficient characteristics than framework-I. It is obvious due to the minimum rate requirement constraint. Unlike framework-I, users in framework-II needs to satisfy minimum rate requirement of users which results in higher bits/channel use and further increases spectral efficiency. 


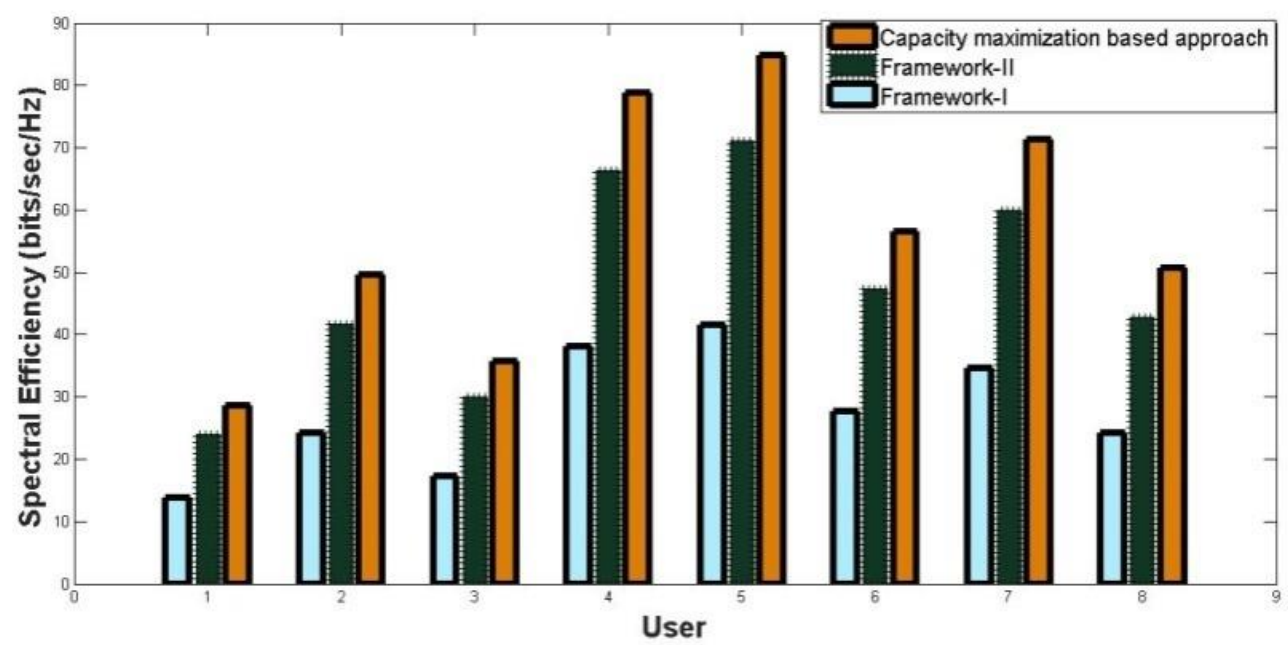

Fig. 11. Spectral efficiency versus users.

Finally, the frameworks are observed in terms of energy efficiency. From Fig. 12 it is observed that, the proposed frameworks are more energy efficient compared to the capacity maximization based approach. Which is obvious due to less power consuming characteristics of the frameworks. Hence, the proposed frameworks of specific QoS constrained resource allocation are very much successful in terms of satisfying total transmit power upper bound of users and energy efficiency compared to conventional capacity maximization based approaches.

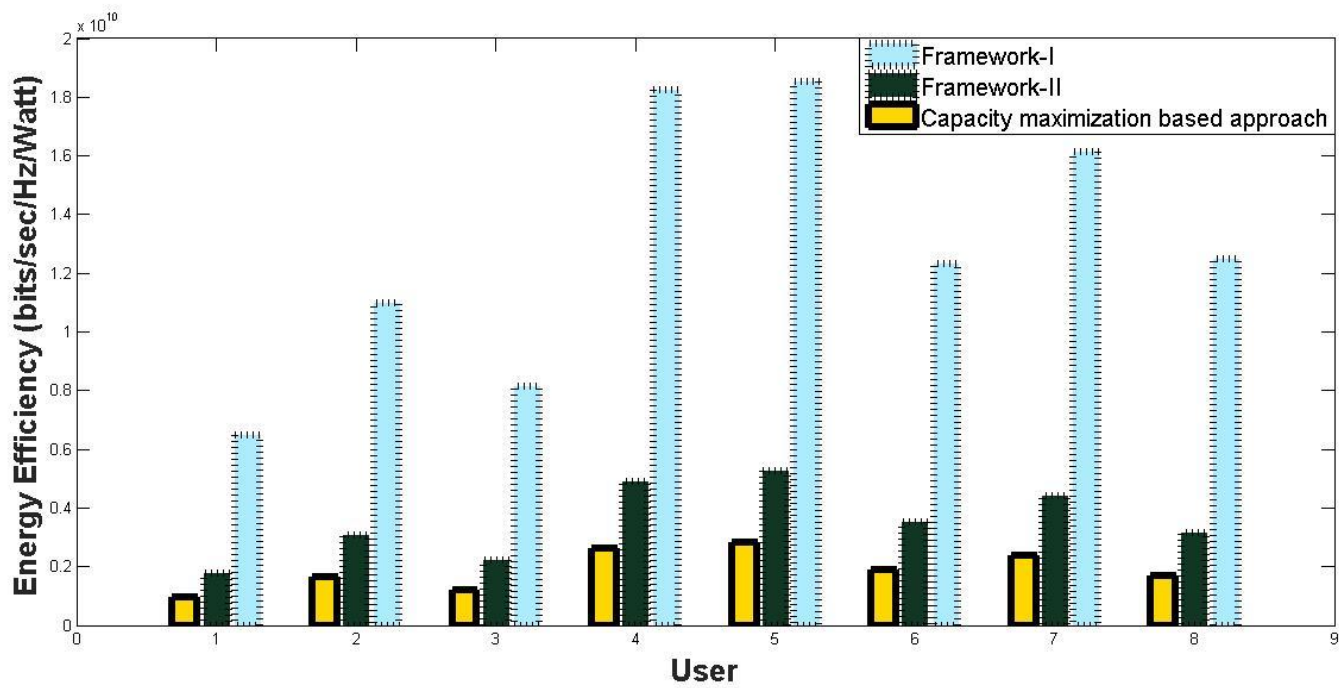

Fig. 12. Energy efficiency versus users.

\section{Conclusion}

In this paper, an uplink cognitive OFDMA system is considered and two frameworks are developed which optimize the transmit power considering SNR threshold and minimum rate requirement as QoS respectively. Simulation results of proposed framework-I illustrate that, more transmit power is required in a subchannel with smaller power gain. Same SNR is obtained across subchannels which is equal to SNR threshold. Higher SNR threshold results in higher total transmit power across users and vice versa. Framework-II shows that, the variation in allocation of transmit power remains almost similar with the change in power gain of the subchannel whereas, unequal SNR is obtained across subchannels. Higher SNR 
is obtained in a subchannel with higher power gain. However, higher value of target BER and users' minimum rate requirement has resulted in higher achieved rate (bits/sec/Hz).Moreover, total transmit power is more when minimum rate requirement of users is higher.

Comparison between the two frameworks and existing capacity maximization based resource allocation framework clearly reveals that, both of the proposed frameworks do not spend all of their power as in existing frameworks based on capacity maximization. Also, framework-II is more spectral efficient than framework-I whereas, framework-I is more energy efficient than framework-II. Both the frameworks are more energy efficient compared to conventional capacity maximization based approaches.

\section{Conflict of Interest}

The authors declare no conflict of interest.

\section{Author Contributions}

All authors contributed equally to the paper and also had approved the final version.

\section{References}

[1] Mitola, J. (2000). Cognitive Radio-An Integrated Agent Architecture for Software Defined Radio.

[2] Chin, W. L., Kao, C. W., \& Le, T. N. (2014, Sept.). Advanced spectrum sensing for ofdm-based cognitive radio networks using cyclic prefix. Proceedings of the 2014 IEEE 80th Vehicular Technology Conference (pp. 1-5).

[3] Jin, S., \& Zhang, X. (2015, March). Compressive spectrum sensing for mimo-ofdm based cognitive radio networks. Proceedings of the 2015 IEEE Wireless Communications and Networking Conference (WCNC) (pp. 2197-2202).

[4] Khumyat, T., \& Uthansakul, P. (2016, June). Channel allocation based on game theory in cr networks over rayleigh fading channel. Proceedings of the $201613^{\text {th }}$ International Conference on Electrical Engineering/Electronics, Computer, Telecommunications and Information Technology (ECTI-CON) (pp. 14).

[5] Sengottuvelan, S., Ansari, J., M"ah"onen, P., Venkatesh, T. G., \& Petrova, M. (2017, May). Channel selection algorithm for cognitive radio networkswith heavy-tailed idle times. IEEE Transactions on Mobile Computing, 16(5), 1258-1271.

[6] Singh, S. K., Kaushik, A., \& Vidyarthi, D. P. (2016, Feb.). A model for cognitive channel allocation using Ga. Proceedings of the 2016 Second International Conference on Computational Intelligence Communication Technology (CICT) (pp. 528-532).

[7] Nabil, M., \& ElNainay, M. (2016, June). Fuzzy-based assignment algorithm for channel sensing task in cognitive radio networks. Proceedings of the 2016 IEEE Symposium on Computers and Communication (ISCC) (pp. 843-848).

[8] Digham, F. F. (2008, March). Joint power and channel allocation for cognitive radios. Proceedings of the 2008 IEEE Wireless Communications and Networking Conference (pp. 882-887).

[9] Cheng, Q., \& Kollimarla, B. (2009, March). Joint channel and power allocation based on user satisfaction for cognitive radio. Proceedings of the 2009 43rd Annual Conference on Information Sciences and Systems (pp. 579-584).

[10] Canales, M., Gallego, J. R., \& Ciria, R. (2011, Sept.). Distributed channel allocation and power control in cognitive radio networks using game theory. Proceedings of the 2011 IEEE Vehicular Technology Conference (VTC Fall) (pp. 1-5).

[11] Zhang, J., Lv, T., Gao, H., \& Lu, Y. (2012, April). Joint uplink power and subchannel allocation in cognitive 
radio network. Proceedings of the 2012 IEEE Wireless Communications and Networking Conference (WCNC) (pp. 306-311).

[12] Li, L., Changqing, X., Pingzhi, F., \& Jian, H. (2014). Resource allocation in orthogonal frequency division multiple access-based cognitive radio systems with minimum rate constraints. International Journal of Communication Systems, 27(8), 1147-1159.

[13] Rawat, D. B., \& Bajracharya, C. (2016, Jan.). Channel and power adaptation for cognitive radios in multiuser ofdm systems. Proceedings of the 2016 IEEE Radio and Wireless Symposium (RWS) (pp. 129132).

[14] Chabalala, C. S., Olst, R. V., \& Takawira, F. (2015, Dec.). Optimal channel selection and power allocation for channel assembling in cognitive radio networks. Proceedings of the 2015 IEEE Global Communications Conference (GLOBECOM) (pp. 1-6).

[15] Shami, N., \& Rasti, M. (2016, April). A joint multi-channel assignment and power control scheme for energy efficiency in cognitive radio networks. Proceedings of the 2016 IEEE Wireless Communications and Networking Conference (pp. 1-6).

[16] Sboui, L., Rezki, Z., \& Alouini, M. S. (2015, Sept.). Energy-efficient power allocation for underlay cognitive radio systems. IEEE Transactions on Cognitive Communications and Networking, 1(3), 273283.

[17] Bedeer, E., Amin, O., Dobre, O. A., Ahmed, M. H., \& Baddour, K. E. (2015, June). Energy-efficient power loading for OFDM-based cognitive radio systems with channel uncertainties. IEEE Transactions on Vehicular Technology, 64(6), 2672-2677.

[18] Wang, S., Shi, W., \& Wang, C. (2015, Sept.). Energy-efficient resource management in OFDM-based cognitive radio networks under channel uncertainty. IEEE Transactions on Communications, 63(9), 3092-3102.

[19] Mao, J., Xie, G., Gao, J., \& Liu, Y. (2013, May). Energy efficiency optimization for OFDM-based cognitive radio systems: A water-filling factor aided search method. IEEE Transactions on Wireless Communications, 12(5), 2366-2375.

[20] Wang, S., Ge, M., \& Zhao, W. (2013, August). Energy-efficient resource allocation for OFDM-based cognitive radio networks. IEEE Transactions on Communications, 61(8), 3181-3191.

[21] Zhou, M., \& Zhao, X. (2016). Energy-efficient power allocation algorithm in cognitive radio networks. IET Communications, 10(17), 2445-2451.

[22] Bepari, D., \& Mitra, D. (2015). Improved power loading scheme for orthogonal frequency division multiplexing based cognitive radio. IET Communications, 9(16), 2033-2040.

[23] Pan, C., Wang, J., Zhang, W., Du, B., \& Chen, M. (2014, Sept.). Power minimization in multi-band multi-antenna cognitive radio networks. IEEE Transactions on Wireless Communications, 13(9), 50565069.

[24] Akter, L., \& Natarajan, B. (2009). Qos constrained resource allocation to secondary users in cognitive radio networks. Computer Communications, 32(18), 1923-1930

[25] Chung, S. T., \& Goldsmith, A. (2001). Degrees of freedom in adaptive modulation: A unified view. Proceedings of the IEEE VTS 53rd Vehicular Technology Conference: Vol. 2. (pp. 1267-1271).

Copyright (C) 2020 by the authors. This is an open access article distributed under the Creative Commons Attribution License which permits unrestricted use, distribution, and reproduction in any medium, provided the original work is properly cited ( $\underline{\text { CC BY 4.0) }}$. 

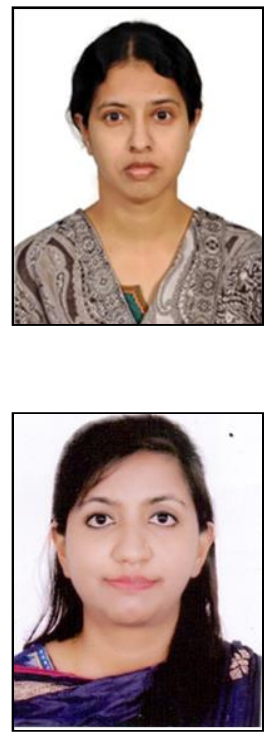

Lutfa Akter received her $\mathrm{PhD}$ degree in electrical engineering from Kansas State University, USA in 2010. She is currently a professor of the Department of Electrical and Electronic Engineering (EEE) of Bangladesh University of Engineering and Technology (BUET). Her current research interests are on resource allocation on cognitive radio networks/wireless sensor networks, wireless sensor deployment, antennas and propagation, localization of wireless video capsule endoscope etc.

Normin Nahar was born in Dhaka, Bangladesh in 1991. She received her B.Sc. degree in electrical, electronic and communication engineering from Military Institute of Science and Technology (MIST), Dhaka, Bangladesh in 2012 and M.Sc. degree in electrical and electronic engineering from Bangladesh University of Engineering and Technology (BUET), Dhaka, Bangladesh in 2019.

After graduation, she joined Bangladesh Power Development Board (BPDB) as an assistant engineer in 2013. Later, she was promoted to sub-divisional engineer in April, 2019. Currently she is working for the Renewable Energy and Research \& Development Department of BPDB. Her research interest mainly lies in the field of wireless communication and signal processing. She received MIST Commandant's and Dean's awards during her undergraduate studies. 